\title{
V. \\ Ein Fall von veralteter Zerreissung der Bänder des medialen Schenkelcondyls im Kniegelenk.
}

\author{
Beobachtet \\ ron \\ Dr. Johann Hönigschmied, \\ kaiserl, und rönigl. Regimentsarzt in Klagenfurt.
}

Im Anschlusse an die von mir im Jahre 1893 in der Deutschen Zeitschrift fur Chirurgie (Bd. XXXVI. S. 587) citirten Fälle von „Zerreissungen der Bänder im Kniegelenk" möge es mir gestattet sein, einen Fall von „veralteter Ruptur des medialen Seiten- und des hinteren Kreuzbandes", welchen ich seither selbst beobachtet habe, kurz anzufübren.

Reserve-Infanterist J. W., welcher bereits im Jahre 1887 seine dreijährige Präsenzdienstzeit beendet hatte und voriges Jahr zur Waffen tibung einruckte, bot bei der ärztlichen Untersuchung folgenden Befund: Der linke Unterschenkel befindet sich beim Gehen in Valgusstellung; in gestreckter Stellung lässt sich derselbe so weit abduciren, dass er mit dem Oberschenkel einen nach aussen offenen Winkel bildet, das Kniegelenk klafft medialwärts, und die Gelenktheile lassen sich umgreifen.

Die Verletzung kam dadurch zu Stande, dass er im Winter 1892, auf einem Schlitten sitzend, mit dem vorgestreckten Unterschenkel mit grosser Kraft an einen Baum anfuhr, wobei der erstere gewaltsam nach aussen gedrängt wurde.

Der Fall ist deshalb von Interesse, weil die Bänderzerreissung ohne eine direct auf das Knie einwirkende Gewalt, durch forcirte Hyperabduction des Unterschenkels za Stande kam.

Zur Begrtindung der Diagnose habe ich auch hier das Experiment an der Leiche herangezogen und bin dabei zu demselben Resultate gelangt, welches ich bereits in meiner oben citirten Arbeit uber die „Wirkung durch gewaltsame Hyperabduction des Unterschenkels" angefulhrt habe: Als ich nämlich an der Leiche eines mittelgrossen, knochenstarken Mannes am linken Kniegelenk das mediale Seitenband durchschnitten hatte, blieb der Unterschenkel mit dem Oberschenkel in gestreckter Stellung noch in Contact; erst als 
ich auch das Ligament. crucial. post. durchtrennt hatte, klaffte das Kniegelenk so weit nach innen, dass die tiefste Stelle der medialen Gelenkfläche der Tibia von dem correspondirenden Punkte des Condylus extern. femoris, etwa $25 \mathrm{Mm}$. weit entfernt war, und man konnte jetzt den Unterschenkel in der Frontalebene so weit nach aussen bewegen, dass er, wie in dem beschriebenen Falle, mit dem Oberschenkel einen nach aussen offenen Winkel bildete.

Das Experiment zeigt deutlich, dass eine Seitenbewegung des Unterschenkels bei gestrecktem Kniegelenk nach aussen, bis zu einem gewissen Grade, bei blosser Zerreissung des medialen Seiten- und des hinteren Kreuzbandes, ausführbar ist. Diese Verletzung wird dann eintreten, wenn die Kraft, welche den Unter-, bezw. den Oberschenkel nach aussen brachte, mit der Zerreissung der genannten Bänder erschöpft ist. Wirkt jedoch die abducirende Gewalt noch weiter fort, dann kommt es auch zur Losreissung des Ligament. cruciat. anterius. 